\title{
Infant Percutaneous Endoscopic Gastrostomy: Risks or Benefits?
}

\author{
Francesco Macchini, Andrea Zanini, Giorgio Farris, Anna Morandi, Giulia Brisighelli, Valerio Gentilino, Giorgio Fava and Ernesto Leva \\ Department of Pediatric Surgery, Fondazione IRCCS Ca' Granda Ospedale Maggiore Policlinico, Milano, Italy
}

Background/Aims: To present a single center's experience with percutaneous endoscopic gastrostomy (PEG) tube placement in infants.

Methods: Clinical records of infants who underwent PEG tube placement between January 2010 and December 2015 were reviewed. All patients underwent an upper gastrointestinal contrast study and an abdominal ultrasonography before the procedure. PEGs were performed with a 6-mm endoscope using the standard pull-through technique. Data regarding gestational age, birth weight, age and weight, days to feeding start, days to full diet, and complications were reviewed.

Results: Twenty-three patients were included. The most common indication was dysphagia related to hypoxic-ischemic encephalopathy. Median gestational age was 37 weeks (range, 24-41) and median birth weight was 2,605 grams (560-4,460). Patients underwent PEG procedures at a median age of 114 days (48-350); mean weight was $5.1 \mathrm{~kg}$ (3.2-8.8). In all patients but one, a 12-Fr tube was positioned. Median feeding start was 3 days (1-5) and on average full diet was achieved 5 days after the procedure (2-11). Six minor complications were recorded and effectively treated in the outpatient clinic; no major complications were recorded.

Conclusions: PEG is safe and feasible in infants when performed by highly experienced physicians. Clin Endosc 2018;51:260-265

Key Words: Percutaneous endoscopic gastrostomy; Infant; Nutrition

\section{INTRODUCTION}

Gastrostomy is indicated in patients needing artificial enteral feeding for a period longer than 2 or 3 weeks. ${ }^{1}$

Indications for gastrostomy in childhood encompass a wide spectrum of conditions, including swallowing disorders, need for fluids and nutritional supplementation, congenital or acquired malformations hindering oral feeding, and children with long-term inadequate food intake. ${ }^{2}$

Aims of enteral tube nutrition in children are to avoid further body weight loss, to correct significant nutritional defi-

Received: August 29, 2017 Revised: October 16, 2017

Accepted: October 27, 2017

Correspondence: Francesco Macchini

Department of Pediatric Surgery, Fondazione IRCCS Ca’ Granda Ospedale Maggiore Policlinico, Via della Commenda 12, Milano 20122, Italy

Tel: +39-02-55032551, Fax: +39-02-55032154

E-mail: francesco.macchini@policlinico.mi.it

(c) This is an Open Access article distributed under the terms of the Creative Commons Attribution Non-Commercial License (http://creativecommons.org/ licenses/by-nc/3.0) which permits unrestricted non-commercial use, distribution, and reproduction in any medium, provided the original work is properly cited. ciencies, to promote growth and improve patients' quality of life, and to provide caregiver satisfaction. ${ }^{3}$

Gastrostomy tubes can be placed using different techniques. In the past, the primary approach was an open surgical gastrostomy. ${ }^{4}$ In 1980, the first percutaneous endoscopic gastrostomy (PEG) procedure was described, and rapidly became the technique of choice both in adults ${ }^{5}$ and in children. ${ }^{6}$ Currently, gastrostomy can also be performed laparoscopically (laparoscopic gastrostomy [LG]), especially in patients with predisposing factors for complications. ${ }^{7}$

To date, only a few papers on the effectiveness and safety of PEG positioning in infancy are available in the literature, 3,8 and evidence is still limited. In addition, some authors report a higher incidence of morbidity and costly complications in PEG as compared to LG. ${ }^{2,9}$

The aim of the present study was to report the experience of a single pediatric surgical center on PEG tube placement in infants. 


\section{MATERIALS AND METHODS}

The clinical data of all children under 1 year of age, who had a PEG placement at our center between January 2010 and December 2015, were analyzed. Indications for PEG placement were based on the European Society for Clinical Nutrition and Metabolism guidelines. ${ }^{1}$ All patients underwent an upper gastrointestinal contrast study and an abdominal ultrasound to exclude contraindications for PEG tube placement, such as intestinal malrotation, situs viscerum inversus, heterotaxy, hepatomegaly, splenomegaly, and ascites. ${ }^{10}$ In presence of these contraindications, a LG was preferred and infants were excluded from the study. When severe gastroesophageal reflux was evident, patients were excluded from the study and were considered candidates for laparoscopic anti-reflux surgery combined with gastrostomy.

PEG tube placement was indicated in infants with no contraindications to general anesthesia, after the exclusion of anatomical intestinal abnormalities or recent abdominal surgery, and after enteral feeding had been well tolerated for at least one month. An informed consent was always obtained from parents.

Gestational age, birth weight, primary and secondary diagnoses, indication for PEG tube placement, age and weight at PEG placement, and complications were recorded. Predisposing factors to major complications, such as multiple system organ failure, cancer, acquired immune deficiency syndrome, and presence of ventriculo-peritoneal shunt, were also studied.

With regards to complications, conversion to open or laparoscopic surgery due to procedural problems, need for prolonged systemic antibiotics, protracted unplanned hospitalization for difficulties with the PEG tube, and the need for surgical intervention during the post-operative period were considered major complications. In particular, hemorrhage, peritonitis, pneumoperitoneum, gastrocolic fistula, and dislocation of the PEG tube represented the most frequent etiopathogenetic factors for major complications. Conversely, peristomal erythema and infection, minor granulomas, and limited leakage were considered minor complications. ${ }^{11,12}$ Time to initial and full gastrostomy feedings was considered. Time to discharge was not considered as a reliable variable due to the severe associated co-morbidities in the treated population that might have influenced this factor. All of the data entered were analyzed with descriptive statistics.

\section{Percutaneous endoscopic gastrostomy procedure}

All procedures were performed in the operating room by two senior pediatric surgeons.

All infants received general anesthesia, with endotracheal intubation due to concomitant co-morbidities and risk of aspiration for swallowing defects. ${ }^{3}$

The standard pull-through technique for PEG positioning described by Gauderer and Stellato ${ }^{5,10}$ was the procedure of choice. First, a standardized abdominal antiseptic skin preparation ( $2 \%$ chlorhexidine $)^{13}$ was applied and prophylactic antibiotics (cefazolin, $40 \mathrm{mg} / \mathrm{kg} / \mathrm{die}$, intravenously) were administered to the patient before and 8 hours following the PEG tube placement; in selected cases (children with allergies or requiring bacterial endocarditis prophylaxis) a more appropriate antibiotic schedule was chosen. ${ }^{14}$ To limit tracheal compression, a neonatal 6-mm outer diameter gastroscope (Pentax Medical, Tokyo, Japan) was introduced through the mouth into the stomach. Inflation was minimized to prevent abdominal distension and to prevent the risk of bowel interposition between the abdominal wall and the stomach. Transillumination of the abdominal wall by the light of the gastroscope was then visualized externally in the epigastrium to guide placement. The PEG placement site was selected between the gastric body and the antrum, preferring a lesser curvature location. ${ }^{15}$ Subsequently, gastric distension was performed by air inflation, driving the anterior gastric wall up against the abdominal wall. $^{7}$

In order to reduce the risk of intestinal perforation during the insertion of the needle, our protocol foresaw insertion using a small needle (22 G, N.12) connected to a syringe filled with a local anesthetic ( $1 \%$ lidocaine, $0.5 \mathrm{~mL} / \mathrm{kg}$ ) passing through the abdominal wall into the stomach. This was accompanied by the application of a gentle aspiration to the syringe. If air was taken in by the syringe during this last maneuver and the tip of the needle was not visible inside the stomach, the risk of an interposing bowel loop was considered high and a different entry-site was chosen. Otherwise, if the tip of the needle was endoscopically identified inside the stomach, the site of the puncture was considered correct, the interposition of the bowel unlikely, and the maneuver feasible. After adequate infiltration of local anesthetic in the subcutaneous tissues a small stab abdominal incision was performed with a number 11 scalpel blade through which a needle, followed by a guide wire, was passed. The wire was then snared by the gastroscope, pulled out in a retrograde fashion from the stomach, through the esophagus, into the mouth, and released. A PEG tube was then tied to this wire, lubricated, and pulled back down through the mouth, esophagus, and stomach, affixing the gastric and abdominal walls together. Either a 12-Fr or a 14-Fr CORFLO-MAX ${ }^{\circledR}$ (Corpak MedSystems, Inc., Buffalo Grove, IL, USA) PEG tube was chosen based on the size of the infant. The gastroscope was then reintroduced to confirm the intragastric position of the inner bumper, and the external bumper was applied to secure 
the tube against the skin. ${ }^{10}$

Post-operative treatment consisted of daily cleansing of the peristomal skin with saline and medication with local antibiotics (gentamycin) for 5 days, followed by a slight loosening of the external bolster after 48 hours and frequent changing of its resting position. Start of feeding and the subsequent feeding program were established by the nutritionist according to the clinical status of the child.

After the first procedure, the PEG is usually left in place between 6 weeks to 6 months. Subsequently, it may be removed indefinitely or, if the child still requires artificial feeding, it may be substituted by balloon gastrostomy. The PEG exchange can be accomplished by 2 methods: (1) percutaneous pull, or (2) endoscopic removal of the PEG tube by grasping and drawing it back. The latter procedure generally requires general anesthesia but may be less traumatic and guarantees an endoscopic confirmation for the correct positioning of the new gastrostomy tube. ${ }^{7.16}$ In our center, the tube is left in place for at least 3 months before removal, preferably using the endoscopic procedure.

\section{RESULTS}

During the time frame considered in the study, of the 91 children who needed gastrostomy in our Institute, 33 (36\%) were infants younger than 1 year of age. Of these, $10(30 \%)$ showed anatomical contraindications to PEG placement; thus, a LG procedure was preferred. In the other 23 infants (70\%), PEG procedures were effectively performed.

Among the PEG group, 14 infants (61\%) were males. The median gestational age at birth was 37 weeks (range, 24-41) and the median birth weight 2,605 grams (range, 560-4,460). Nine newborns (39\%) were premature (gestational age $<37$ weeks). Patients underwent PEG positioning at a median age of 114 days (range, 48-350), median weight at the time of operation was 5,100 grams (range, 3,200-8,800).

The primary indication was dysphagia or inadequate swallowing. Secondary diagnoses were neurologic disorders due to hypoxic-ischemic encephalopathy at birth in 11 (48\%), plurimalformative syndromes in $4(16 \%)$, giant neck lymphangioma in $4(16 \%)$, metabolic disorders in $1(4 \%)$, chronic lung disease in $1(4 \%)$, myasthenia gravis in $1(4 \%)$, and intra-ventricular hemorrhage in 1 (4\%).

None of the infants studied presented predisposing factors to major complications, such as multiple system organ failure, cancer, and acquired immune deficiency syndrome, and none had a ventriculo-peritoneal shunt. Gastroesophageal reflux disease (GERD and other major intestinal malformations were excluded before surgery.
A 12-Fr tube was positioned in all patients, with the exception of one infant where a 14-Fr tube was preferred due to his weight at the time of surgery. No procedure needed to be converted to open or laparoscopic surgery. The mean duration of the procedure was 16 minutes (range, 14-25 minutes; median, 15 minutes).

The mean time to initial feeding was 2 days (range, 1-5 days; median, 3 days), and the mean time to full feeding (100 $\mathrm{kcal} / \mathrm{kg}$ ) was 5 days (range, 2-11 days; median, 7.5 days).

Six (26\%) minor complications were recorded (3 peristomal hyperemia, 2 granulomas, 1 leakage) and were treated in the outpatient clinic with local cleansing with saline and application of $2 \%$ eosine; complete resolution was achieved within a few weeks.

No major complications were recorded at follow-up (mean, 34 months; range, 8-62 months) in our series.

PEG tubes were endoscopically substituted by balloon-buttons in 20 patients after a mean period of 5 months (range, 3-8 months), while 3 children had the PEG tube definitively removed before its substitution. No complications were recorded during or after these maneuvers. Twelve patients had the device definitively removed after a mean of 15 months from placement, while in 11 patients the tube is currently in use. The stoma channel spontaneously closed in all patients after local medications and no child needed surgical closure of the gastrostomy site.

\section{DISCUSSION}

Oro- or nasogastric feeding is used for enteral nutrition in neonates and infants with insufficient oral food intake. However, due to the risk of tube dislodgement and aspiration, many neonatologists and pediatricians do not feel comfortable in discharging these infants, and thus prolong their hospitalization. $^{3,17-19}$

Gastrostomy represents a more stable and a safer tool to insure long-term enteral nutrition. It is usually well tolerated and guarantees a better quality of life compared to parenteral and nasogastric feeding. ${ }^{20}$ Among the different gastrostomy procedures, the PEG technique has proven to be more cost-effective and safer than surgical gastrostomy since $1980 .{ }^{5}$ In fact, it does not require laparotomy, it minimizes pain, and requires a shorter hospital stay. PEG placement was calculated to take on average much less time than surgical gastrostomy (15 minutes vs. 35 minutes $)^{1,21}$ or LG (15 minutes vs. 48 minutes). ${ }^{22}$

In our population, the time required for performing a PEG procedure was 16 minutes. In addition, PEG is also generally associated with a shorter hospital stay since it can be used earlier than surgical or LG. ${ }^{9}$ In our series, infants achieved full 
feeds in 5 days on average.

At present, there is still a lack of evidence on the safety of PEG placement in infants. In addition, it is difficult to compare papers on PEG placement in children younger than 1 year of age because in most studies the population evaluated was mixed with older children. ${ }^{3}$

In our experience, PEG placement resulted effective in all 23 infants who underwent the procedure. As recommended by our previous experience, the pull technique was the procedure of choice in our population. The use of a neonatal endoscope appeared effective in reducing the invasiveness of the procedure in neonates and infants, thus we recommend its use in infants.

Some recent publications stress the higher incidence of morbidity and costly complications in PEG as compared to LG procedures. ${ }^{2.9}$ In contrast, in our series we experienced complications in only $26 \%$ of patients and all were minor. It is difficult to compare our data with the literature where overall complication rates between $4 \%$ and $43 \%$ have been reported in the pediatric population. ${ }^{23,24}$ This range in rate of observed complications is so broad likely because these studies report very different types of complications. For example, erythe$\mathrm{ma}$ and granuloma are regarded as complications by some authors, but not by others. ${ }^{25}$ While, some authors consider multiple system organ failure, cancer, and acquired immune deficiency syndrome as predisposing factors for major complications of PEG procedures. ${ }^{26}$ Vervloessem et al. ${ }^{27}$ reported that children with a ventricular peritoneal shunt had a higher incidence of major complications. In contrast other authors did not consider these as a major contraindication to PEG. ${ }^{28}$ Furthermore, some physicians believe that PEG positioning may predispose to GERD but a strict etiopathogenetic correlation has still to be proven. ${ }^{23,24}$ In our series, none of the infants presented the abovementioned predisposing factors for major complications of PEG tube placement. With regards to GERD, it was never observed in our patients after PEG placement. The possible explanation of our findings may lie in the fact that, since we considered GERD as a contraindication, it was always ruled out before performing PEG placement and, when present, we would combine it with a laparoscopic anti-reflux procedure. Furthermore, the incidence of postoperative GERD may have been decreased by positioning the PEG tube in a lesser curvature location, rather than on the greater curvature of the stomach between the gastric body and the antrum as suggested in a previous study by Seekri et al. ${ }^{15}$ It is likely that placing the PEG distally into the stomach may reduce the traction on the fundus, preserving the His angle and thus avoid a risk for GERD development.

With regards to the surgical technique, the incidence of major complications in children is reported to be between
$0.5 \%$ and $17 \%$ after PEG placement and $0 \%$ and $4 \%$ following LG. $^{29}$ In particular, a recent paper reported an incidence of gastro-colic fistula in 3.8\% of PEG and 0\% of LG procedures and of early tube dislodgement in $7.6 \%$ of PEG and in $0 \%$ of LG procedures performed in infants. The subsequent increase of costs for the treatment of complications led authors to conclude that infants undergoing PEG have more morbidity and costly complications and that LG may be the less burdensome approach to gastrostomy. ${ }^{9}$ Our experience does not seem to reproduce the results of the previous cited study as no major complications were recorded. With regards to minor complications, these are usually easy to treat and do not denote any clinical significance or discomfort for children and parents. In this series, no child experienced respiratory problems related to the procedure. Indeed, in small infants the use of a neonatal endoscope (6-mm diameter) under general anesthesia with endotracheal intubation may have helped to avoid tracheal compression by the endoscope and insufflations of the stomach thus preventing onset of respiratory issues. ${ }^{2}$

One of the factors explaining the low rate of complications may be related to the presence of dedicated experienced surgeons at our Institute. In fact, as suggested in the literature, ${ }^{30}$ surgical experience is an important factor in reducing significant complications and PEG should be performed only by specialists who have received appropriate training and supervision. Vervloessem et al. ${ }^{27}$ reported a significant decrease in the rate of major complications with the increase in operator experience. Of course, technical skills are correlated to the number of procedures performed..$^{30}$ As a consequence, to achieve an adequate amount of experience; it is crucial that the physician who performs the PEG procedure in infants also regularly does so in an older pediatric population. In our study group, all procedures were performed by the same gastro-intestinal team of surgeons, who were highly experienced in pediatric endoscopy $(>5,000$ pediatric endoscopic examinations).

Another factor that may have limited the complication rate could be represented by the relatively limited series of patients evaluated in this study. Indeed, the homogeneity of the clini$\mathrm{cal}$ and surgical data recorded over a relatively short period of time, as well as the relatively high number of infants treated compared to experience reported from other Italian institutions in the general pediatric population, ${ }^{31}$ may represent the main strengths of this work.

The third and probably most significant factor is represented by the strict compliance to the PEG placement technique described above. This may represent a significant safety factor. Finally, the routine preoperative intestinal study consisting of ultrasonography and a complete gastrointestinal contrast study to exclude anatomical contraindications appeared to be 
useful in preventing potentially severe complications. ${ }^{10}$ In our experience, in presence of these contraindications, despite its higher invasiveness, we believe that LG is a safer procedure. Due to the clinical differences between the LG and PEG groups, they were not considered comparable.

With regards to the substitution of the PEG tube, some papers have recorded incidences of stomal disruption following traction removal. ${ }^{716}$ Currently, there is no evidence on the safe timing of traction removal available and complications have been described even months or years after insertion. According to our experience, a minimum of 3 months before removing or exchanging the tube may represent a safety factor. To date, we have removed all of the original PEG tubes in our series of infants, and no complications have been encountered. Replacing the PEG tube with a balloon gastrostomy button is a routine procedure for our gastro-intestinal team of surgeons, and they prefer an equal or smaller diameter device in order to avoid any trauma during the procedure. Accordingly, we avoid dilating the stoma during the substitution procedure in order to reduce the risk of gastric wall disconnection. ${ }^{3}$

The main limitation of the present study is represented by the fact that it is a retrospective study on a relatively small population. However, considering the paucity of infants requiring full enteral nutrition and the short study period, we believe that this may represent only a minor limitation.

In conclusion, PEG tube placement seems to guarantee more benefits than risks in infancy, revealing at present to be a procedure with the lowest grade of invasiveness. When performed by expert surgeons, it is safe and effective also in infancy. On the contrary, LG may represent a valid option to avoid complications in selected cases.

\section{Conflicts of Interest}

The authors have no financial conflicts of interest.

\section{REFERENCES}

1. Loser C, Aschl G, Hébuterne X, et al. ESPEN guidelines on artificial enteral nutrition--percutaneous endoscopic gastrostomy (PEG). Clin Nutr 2005;24:848-861.

2. Merli L, De Marco EA, Fedele C, et al. Gastrostomy placement in children: percutaneous endoscopic gastrostomy or laparoscopic gastrostomy? Surg Laparosc Endosc Percutan Tech 2016;26:381-384.

3. Wilson L, Oliva-Hemker M. Percutaneous endoscopic gastrostomy in small medically complex infants. Endoscopy 2001;33:433-436.

4. Sulkowski JP, De Roo AC, Nielsen J, et al. A comparison of pediatric gastrostomy tube placement techniques. Pediatr Surg Int 2016;32:269275.

5. Gauderer MW, Ponsky JL, Izant RJ Jr. Gastrostomy without laparotomy: a percutaneous endoscopic technique. J Pediatr Surg 1980;15:872-875.

6. Viktorsdottir MB, Óskarsson K, Gunnarsdóttir A, Sigurdsson L. Percutaneous endoscopic gastrostomy in children: a population-based study from iceland, 1999-2010. J Laparoendosc Adv Surg Tech A 2015;25:248-
251.

7. Minar P, Garland J, Martinez A, Werlin S. Safety of percutaneous endoscopic gastrostomy in medically complicated infants. J Pediatr Gastroenterol Nutr 2011;53:293-295.

8. ASGE Standards of Practice Committee, Lightdale JR, Acosta R, et al Modifications in endoscopic practice for pediatric patients. Gastrointest Endosc 2014;79:699-710.

9. Landisch RM, Colwell RC, Densmore JC. Infant gastrostomy outcomes: the cost of complications. J Pediatr Surg 2016;51:1976-1982.

10. Gauderer MWL, Stellato TA. Percutaneous endoscopic gastrostomy in children: the technique in detail. Pediatr Surg Int 1991;6:82-87.

11. McSweeney ME, Jiang H, Deutsch AJ, Atmadja M, Lightdale JR. Longterm outcomes of infants and children undergoing percutaneous endoscopy gastrostomy tube placement. J Pediatr Gastroenterol Nutr 2013;57:663-667.

12. McSweeney ME, Kerr J, Jiang H, Lightdale JR. Risk factors for complications in infants and children with percutaneous endoscopic gastrostomy tubes. J Pediatr 2015;166:1514-1519.e1.

13. Sathiyamurthy S, Banerjee J, Godambe SV. Antiseptic use in the neonatal intensive care unit - a dilemma in clinical practice: an evidence based review. World J Clin Pediatr 2016;5:159-171.

14. Jafri NS, Mahid SS, Minor KS, Idstein SR, Hornung CA, Galandiuk S. Meta-analysis: antibiotic prophylaxis to prevent peristomal infection following percutaneous endoscopic gastrostomy. Aliment Pharmacol Ther 2007;25:647-656.

15. Seekri IK, Rescorla FJ, Canal DF, Zollinger TW, Saywell R Jr, Grosfeld JL. Lesser curvature gastrostomy reduces the incidence of postoperative gastroesophageal reflux. J Pediatr Surg 1991;26:982-984; discussion 984985.

16. Fox VL, Abel SD, Malas S, Duggan C, Leichtner AM. Complications following percutaneous endoscopic gastrostomy and subsequent catheter replacement in children and young adults. Gastrointest Endosc 1997;45:64-71.

17. Sporik R. Why block a small hole? The adverse effects of nasogastric tubes. Arch Dis Child 1994;71:393-394.

18. Greenspan JS, Wolfson MR, Holt WJ, Shaffer TH. Neonatal gastric intubation: differential respiratory effects between nasogastric and orogastric tubes. Pediatr Pulmonol 1990;8:254-258.

19. Raddish M, Merritt TA. Early discharge of premature infants. A critical analysis. Clin Perinatol 1998;25:499-520.

20. Baker L, Beres AL, Baird R. A systematic review and meta-analysis of gastrostomy insertion techniques in children. J Pediatr Surg 2015;50:718725 .

21. Ljungdahl M, Sundbom M. Complication rate lower after percutaneous endoscopic gastrostomy than after surgical gastrostomy: a prospective, randomized trial. Surg Endosc 2006;20:1248-1251.

22. Jones VS, La Hei ER, Shun A. Laparoscopic gastrostomy: the preferred method of gastrostomy in children. Pediatr Surg Int 2007;23:1085-1089.

23. Behrens R, Lang T, Muschweck H, Richter T, Hofbeck M. Percutaneous endoscopic gastrostomy in children and adolescents. J Pediatr Gastroenterol Nutr 1997;25:487-491.

24. Khattak IU, Kimber C, Kiely EM, Spitz L. Percutaneous endoscopic gastrostomy in paediatric practice: complications and outcome. J Pediatr Surg 1998;33:67-72.

25. Kutiyanawala MA, Hussain A, Johnstone JM, Everson NW, Nour S. Gastrostomy complications in infants and children. Ann R Coll Surg Engl 1998;80:240-243.

26. Davidson PM, Catto-Smith AG, Beasley SW. Technique and complications of percutaneous endoscopic gastrostomy in children. Aust N Z J Surg 1995;65:194-196.

27. Vervloessem D, van Leersum F, Boer D, et al. Percutaneous endoscopic gastrostomy (PEG) in children is not a minor procedure: risk factors for major complications. Semin Pediatr Surg 2009;18:93-97.

28. Oterdoom LH, Marinus Oterdoom DL, Ket JCF, van Dijk JMC, Scholten P. Systematic review of ventricular peritoneal shunt and percutaneous 
endoscopic gastrostomy: a safe combination. J Neurosurg 2017;127:899904.

29. McSweeney ME, Smithers CJ. Advances in pediatric gastrostomy placement. Gastrointest Endosc Clin N Am 2016;26:169-185.

30. Hassall E. Requirements for training to ensure competence of endoscopists performing invasive procedures in children. Training and education committee of the North American society for pediatric gas- troenterology and nutrition (NASPGN), the ad hoc pediatric committee of American society for gastrointestinal endoscopy (ASGE), and the executive council of NASPGN. J Pediatr Gastroenterol Nutr 1997;24:345347.

31. Fascetti-Leon F, Gamba P, Dall'Oglio L, et al. Complications of percutaneous endoscopic gastrostomy in children: results of an Italian multicenter observational study. Dig Liver Dis 2012;44:655-659. 